\title{
The Use of a Green Fluorescent Protein Porcine Model to Evaluate Host Tissue Integration into Extracellular Matrix Derived Bionanocomposite Scaffolds
}

\author{
S. E. Smith, ${ }^{1}$ R. A. White, ${ }^{1,2}$ D. A. Grant, ${ }^{1}$ and S. A. Grant ${ }^{1}$ \\ ${ }^{1}$ Department of Bioengineering, University of Missouri, 254 Agricultural Engineering, Columbia, MO 65211, USA \\ ${ }^{2}$ Department of Orthopaedic Surgery, University of Missouri, Missouri Orthopaedic Institute, 1100 Virginia Avenue Columbia, \\ MO 65212, USA \\ Correspondence should be addressed to S. A. Grant; grantsa@missouri.edu
}

Received 30 September 2014; Accepted 10 December 2014

Academic Editor: Kimimasa Tobita

Copyright (C) 2015 S. E. Smith et al. This is an open access article distributed under the Creative Commons Attribution License, which permits unrestricted use, distribution, and reproduction in any medium, provided the original work is properly cited.

\begin{abstract}
When using heterogeneous extracellular matrix (ECM) derived scaffolds for soft tissue repair, current methods of in vivo evaluation can fail to provide a clear distinction between host collagen and implanted scaffolds making it difficult to assess host tissue integration and remodeling. The purpose of this study is both to evaluate novel scaffolds conjugated with nanoparticles for host tissue integration and biocompatibility and to assess green fluorescent protein (GFP) expressing swine as a new animal model to evaluate soft tissue repair materials. Human-derived graft materials conjugated with nanoparticles were subcutaneously implanted into GFP expressing swine to be evaluated for biocompatibility and tissue integration through histological scoring and confocal imaging. Histological scoring indicates biocompatibility and remodeling of the scaffolds with and without nanoparticles at 1,3 , and 6 months. Confocal microscope images display host tissue integration into scaffolds although nonspecificity of GFP does not allow for quantification of integration. However, the confocal images do allow for spatial observation of host tissue migration into the scaffolds at different depths of penetration. The study concludes that the nanoparticle scaffolds are biocompatible and promote integration and that the use of GFP expressing swine can aid in visualizing the scaffold/host interface and host cell/tissue migration.
\end{abstract}

\section{Introduction}

The use of naturally derived biomaterials has become of increasing interest due to their superior performance over traditionally used synthetic materials [1]. Natural scaffolds materials are often derived from the extracellular matrix (ECM) that may help to evade the negative foreign body reaction of synthetic materials due to their biocompatible components. Additionally, natural materials have been shown to release growth factors as they degrade, promoting cellular attachment and remodeling [2]. These types of scaffolds are often used in many surgical procedures including tendon and ligament repair [3], hernia repair [4], wound healing [5], and other soft tissue repairs [6].

With an increasing amount of research on soft tissue repair materials comes the increased demand for better techniques to assess their in vivo performance. New ECM deposition as part of the tissue remodeling process is a key characteristic in the assessment of soft tissue repair materials. The current standard of in vivo evaluation is H\&E and Masson's trichrome histology staining. When using ECM derived materials, there is not always a clear distinction between the host tissue and the tissue scaffold. If the components of the host tissue and scaffold material are very similar, they may appear similar when stained making small differences difficult to decipher. This difficulty is especially true of larger, heterogeneous tissues such as the anterior tibialis tendon. Immunohistochemistry is another standard technique used $[7,8]$ although the problem arises that most biomarkers such as collagen [9] that are present on the scaffold are also present in the host tissue preventing distinction between the host tissue and scaffold. Another issue when utilizing fluorescence technology for visualization is naturally occurring autofluorescence of the scaffold as well as fixative-induced 
autofluorescence which can interfere with or even mask the fluorescent signal [10, 11].

Alternative methods to assess soft tissue integration, such as X-ray diffraction enhanced imaging [12], MRI [13], and megavoltage cone beam CT [14], fail to provide data specific enough to assess integration. Correlative light microcopy has potential but does not allow for visualization on a scale large enough for scaffolds implanted into an animal model [15]. An improved method for visualization of host tissue integration will allow for the distinction between host tissue and scaffold on a size scale comparable to histology to aid in the assessment of biocompatibility and tissue integration in an in vivo model.

There has been recent development in the use of fluorescence imaging techniques to address the need for better visualization of host tissue integration. One of these techniques involves GFP expressing animals such as mice and swine [16]. GFP expressing animals have a GFP jellyfish gene inserted into its genome which produces GFP that will fluoresce green under incident blue wavelengths of light [17]. There are multiple studies that involve injecting cells from GFP animals into standard animal models to assess the remodeling behavior of the injected cells and the host response [1820]. While this technique allows for distinction between host tissue and scaffolds, it does not allow for natural remodeling by host tissue cells that would occur when scaffolds are implanted without cellular augmentation.

The scaffolds used in this study are human-derived grafts conjugated with gold nanoparticles (AuNP) and hydroxyapatite nanoparticles (nano-HAp). Nanoparticles are utilized in order to produce a functionally graded scaffold that contains AuNP immobilized throughout the graft with nano-HAp immobilized on the ends of the graft which may serve as an improved anterior cruciate ligament (ACL) repair material. Nano-HAp has been shown to enhance osteoblast attachment, osteoconductivity, and biocompatibility [21]. The addition of hydroxyapatite nanoparticles (nano-HAp) to scaffolds may also improve integration at the bone-ligament interface [22]. It has been shown that the addition of gold nanoparticles (AuNP) to collagen based scaffolds improves in vivo degradation resistance possibly due to the hindering of collagenase binding sites by the AuNP [23]. Additional potential benefits of adding AuNP to tissue include antimicrobial effects [24-26], free radical scavenging [27, 28], enhanced cellular attachment [29], and remodeling [30]. Further design and development of nanocomposite scaffolds are established elsewhere and will not be addressed further [23, 30-35].

The purpose of this study is both to evaluate novel scaffolds conjugated with nanoparticles for host tissue integration and biocompatibility and to assess GFP expressing swine as a new animal model to evaluate soft tissue repair materials. Nanocomposite scaffolds were implanted subcutaneously into GFP expressing swine. At 1-, 3-, and 6-month time points, scaffolds were explanted and histological scoring was performed for cellular infiltration, multinucleated giant cells (MNGC), vascularity, connective tissue organization, fibrous encapsulation, and scaffold degradation. Confocal microscopy was performed on explanted samples to visualize the scaffold/tissue interface. It is hypothesized that the use of
GFP swine will aid in visualization of fluorescent host tissue infiltrating into the nonfluorescent scaffold. In addition, it is hypothesized that the addition of nanoparticles to scaffolds will enhance biocompatibility and tissue remodeling.

\section{Methods}

2.1. Experimental Design. All animal procedures were approved by the University of Missouri Institutional Animal Care and Use Committee. GFP expressing swine were obtained from the National Swine Resource and Research Center (Columbia, MO). Decellularized human anterior tibialis tendons from the Musculoskeletal Transplant Foundation were used as scaffolds (Edison, NJ). Scaffolds types were AuNP, AuNP + nano-HAp, or "crosslinked" which are scaffolds that have undergone the cross-linking process without nanoparticles added. Scaffolds with nano-HAp alone were not evaluated due to limitations of the size of the study and due to the fact that the future ACL application of the scaffolds would not require nano-HAp alone. Scaffold sizes varied between $1 \times 3 \mathrm{~cm}$ and $2.5 \times 3 \mathrm{~cm}$. One scaffold of each type was implanted subcutaneously into 12 swine with the placement of the type of scaffold randomized within each animal. Scaffolds were bilaterally implanted and equally spaced across the abdomen. Each corner of the scaffold was sutured to the fascia layer. At the end of each time point of 1,3 , or 6 months, 4 swine were euthanized. Scaffolds and surrounding tissue were explanted and submitted for histological or confocal microscopy analysis.

2.2. Cross-Linking and Sterilization. Tissue was stored at $-20^{\circ} \mathrm{C}$ until ready to use. Tissues were cross-linked according to a protocol previously established by Deeken et al. [32]. Tissues were incubated for $15 \mathrm{~min}$ in a room temperature solution comprised of 50:50 (v/v) solution of acetone and $1 \mathrm{x}$ phosphate buffered saline (PBS) $(\mathrm{pH}=7.5)$ with $2 \mathrm{mM}$ 1-ethyl3-[3-dimethylaminopropyl] carbodiimide (EDC) and $5 \mathrm{mM}$ N-hydroxysuccinimide (NHS). NHS was added to a small amount of dimethylformamide (DMF) and EDC was added to a small amount of 0.1 M 2-(N-morpholino) ethanesulfonic acid (MES) in $0.5 \mathrm{M}$ sodium chloride $(\mathrm{NaCl})(\mathrm{pH}=6.0)$ before being combined and added to the acetone and PBS solution. AuNP $\left(5.6 \times 10^{9}\right.$ particles $\left./ \mathrm{mL}\right)$ were functionalized with $15 \mu \mathrm{M} 2$-mercaptoethylamine (MEA) in water. NanoHAp solution $(1 \% \mathrm{w} / \mathrm{v})$ was sonicated at $45^{\circ} \mathrm{C}$ prior to being added to tissue to avoid aggregation. Each $1 \times 3 \mathrm{~cm}$ to $2.5 \times$ $3 \mathrm{~cm}$ piece of tissue received $1 \mathrm{~mL}$ of nanoparticle colloid. Scaffolds with both AuNP and nano-HAp received $0.5 \mathrm{~mL}$ of each colloid simultaneously. Scaffolds without nanoparticles received an additional $1 \mathrm{~mL}$ of cross-linking solution. After $24 \mathrm{~h}$, scaffolds were rinsed twice in 1x PBS for $24 \mathrm{~h}$ each. Scaffolds were sterilized in a solution of $1 \mathrm{M} \mathrm{NaCl}$ and $0.1 \%$ $(\mathrm{v} / \mathrm{v})$ peracetic acid for $30 \mathrm{~min}$ according to protocol by Deeken et al. [31]. Scaffolds were rinsed twice in sterile 1x PBS for $24 \mathrm{~h}$ each.

2.3. Histological Preparation. Explanted samples for histological analysis were stored in 10\% neutral buffered formalin. Excess tissue was removed from samples before being 
TABLE 1: Qualitative and semiquantitative scoring criteria. Quantitative score is per 400x field.

\begin{tabular}{|c|c|c|c|c|}
\hline Criteria & 0 & 1 & 2 & 3 \\
\hline Cellular infiltration & $0-50$ cells & 51 and 100 cells & $101-150$ cells & $>150$ cells \\
\hline $\begin{array}{l}\text { Multinucleated giant } \\
\text { cells (MNGC) }\end{array}$ & 0 MNGC & 1-2 MNGC & 3-4 MNGC & $>5$ MNGC \\
\hline Vascularity & $\begin{array}{c}\text { Either } 0 \text { or } 1 \text { blood } \\
\text { vessel }\end{array}$ & $2-5$ blood vessels & 6-10 blood vessels & $>10$ blood vessels \\
\hline $\begin{array}{l}\text { Connective tissue } \\
\text { organization }\end{array}$ & $\begin{array}{l}\text { Original scaffold is } \\
\text { intact }\end{array}$ & $\begin{array}{l}\text { Original scaffold disrupted; } \\
\text { poorly organized new host } \\
\text { ECM present }\end{array}$ & $\begin{array}{c}\text { Mixture of highly organized and } \\
\text { poorly organized connective } \\
\text { tissue }\end{array}$ & $\begin{array}{l}\text { Highly organized } \\
\text { connective tissue present }\end{array}$ \\
\hline Fibrous encapsulation & $\begin{array}{l}\text { No fibrous } \\
\text { encapsulation }\end{array}$ & $\begin{array}{l}\text { Less than } 50 \% \text { of the periphery } \\
\text { has fibrous encapsulation }\end{array}$ & $\begin{array}{l}50 \%-90 \% \text { of the periphery has } \\
\text { fibrous encapsulation }\end{array}$ & $\begin{array}{l}\text { Complete fibrous } \\
\text { encapsulation }\end{array}$ \\
\hline Scaffold degradation & Scaffold is intact & $\begin{array}{l}\text { Scaffold fibers are disrupted } \\
\text { and present in groups or large } \\
\text { bundles }\end{array}$ & $\begin{array}{c}\text { Scaffold fibers are disrupted and } \\
\text { present in single fibers or small } \\
\text { bundles }\end{array}$ & No scaffold fibers remain \\
\hline
\end{tabular}

embedded in paraffin wax. Sections of $5 \mu \mathrm{m}$ thickness were cut, mounted on glass slides, and stained with hematoxylin and eosin (H\&E). Control samples were identically treated.

2.4. Histological Scoring. The semiquantitative grading scale used for histological scoring originates from studies by Grant et al. $[30,36]$. The scoring guide is presented in Table 1 . The following criteria were scored: cellular infiltration, multinucleated giant cells (MNGC), vascularity, connective tissue organization, fibrous encapsulation, and scaffold degradation. For cellular infiltration, MNGC, and vascularity, 10 equidistant sites along the scaffold/host interface edge were scored and averaged. Connective tissue organization, fibrous encapsulation, and scaffold degradation were qualitatively assessed as a whole scaffold. Some scaffolds had fewer than 10 sites scored because not enough tissue was present. Slides were evaluated by an external veterinary pathologist.

2.5. Confocal Imaging. A small portion of explanted scaffolds were retained for confocal microscopy. These explants were immediately frozen on dry ice after harvest and stored at $-20^{\circ} \mathrm{C}$ until imaged. When ready to image, explants were placed in an imaging specimen holder with 1x PBS. Immediately after scaffolds were thawed, images were taken using a Zeiss LSM 510 META confocal microscope (Oberkochen, Germany) using a $488 \mathrm{~nm}$ laser excitation and 500-550 nm emission filter. All images were taken using a Plan-Neofluar $10 \mathrm{x} / 0.3$ objective or Plan-Apochromat 20x/0.75 objective. Imaging parameters such as laser power were optimized to specific images to visualize features. Images were individually adjusted for brightness and contrast using Zeiss LSM Image Browser software.

2.6. Statistical Analysis. All statistical analyses were performed using SAS 9.3 software (Cary, NC). One-way analysis of variance with a Tukey posttest was conducted to determine significant differences between means. Student's $t$-test was used to determine if select score means were significantly different than zero. Each time point and category began with $N=4$ but some samples could not be recovered or scored resulting in some groups having final $N$ values at 3 or below that could not be analyzed by a statistical test. Means that are not accompanied by the standard error of the mean have only one score for that category. Significance was set at $P<0.05$.

\section{Results}

Control histology images of the scaffold before implantation are shown in Figure 1. The heterogeneity of the cross-linked human anterior tibialis tendon derived scaffold can be seen between the proximal end (Figure 1(a)) and the distal end (Figure 1(b)). Tendon fibers are mostly uniaxially aligned at the proximal end and appear less organized at the distal end. The absence of human cells should also be noted confirming that the scaffolds were fully decellularized.

Semiquantitative and qualitative scoring results are presented in Table 2 and representative images are shown in Figure 2. Scores are presented as mean score \pm standard error of the mean for each scoring criterion at 1,3, and 6 months after implantation.

3.1. Semiquantitative Histology. Scores for cellular infiltration do not show any statistical significant differences between groups or time points. Scaffolds at 1 month are mild to moderately infiltrated with mononuclear cells and peripheralforming cell aggregates. Scaffolds at 3 months also exhibit mild to moderate infiltration of mononuclear cells with clusters of cells in the center and periphery of the scaffold. At 6 months mononuclear cell infiltration is moderate to marked in the center and periphery with many nuclei counted being fibroblasts and not inflammatory cells.

Scores for MNGC are not statistically different between groups or time points. The score for each group at each time point is not statistically different than zero. Very few MNGC are seen for any group with individual scores of less than 0.5 and averages below 0.2. All cross-linked scaffolds at 3 months, AuNP scaffolds at 6 months, and AuNP + nano-HAp scaffolds at 1 month have scores of zero.

Cross-linked scaffolds have significantly higher vascularity scores at 6 months compared to 3-month and 1-month scaffolds. AuNP scaffolds have significantly higher vascularity scores at 6 months compared to 1 month. Although 
TABLE 2: Qualitative and quantitative histological scoring results.

\begin{tabular}{|c|c|c|c|c|c|c|}
\hline Scaffold type & $\begin{array}{l}\text { Cellular } \\
\text { infiltration }\end{array}$ & $\begin{array}{l}\text { Multinucleated giant } \\
\text { cells (MNGC) }\end{array}$ & Vascularity & $\begin{array}{c}\text { Connective tissue } \\
\text { organization }\end{array}$ & $\begin{array}{c}\text { Fibrous } \\
\text { encapsulation }\end{array}$ & $\begin{array}{c}\text { Scaffold } \\
\text { degradation }\end{array}$ \\
\hline \multicolumn{7}{|l|}{ Cross-linked } \\
\hline 1 month & $2.10 \pm 0.49$ & $0.03 \pm 0.03$ & $0.23 \pm 0.12$ & $1.00 \pm 0.00$ & $1.33 \pm 0.33$ & $1.00 \pm 0.00$ \\
\hline 3 months & $2.00 \pm 0.71$ & $0.00 \pm 0.00$ & $0.40 \pm 0.15$ & $1.33 \pm 0.33$ & 2.00 & $1.00 \pm 0.00$ \\
\hline 6 months & $2.38 \pm 0.38$ & $0.15 \pm 0.10$ & $1.13 \pm 0.21$ & $2.00 \pm 0.58$ & - & $1.5 \pm 0.29$ \\
\hline \multicolumn{7}{|l|}{ AuNP } \\
\hline 1 month & $1.67 \pm 0.34$ & $0.07 \pm 0.03$ & $0.03 \pm 0.07$ & $1.00 \pm 0.00$ & $2.00 \pm 0.00$ & $1.00 \pm 0.00$ \\
\hline 3 months & $1.13 \pm 0.52$ & $0.20 \pm 0.20$ & $0.40 \pm 0.12$ & $1.33 \pm 0.33$ & $2.00 \pm 0.00$ & $1.00 \pm 0.00$ \\
\hline 6 months & $2.46 \pm 0.33$ & $0.00 \pm 0.00$ & $1.27 \pm 0.43$ & $2.00 \pm 0.00$ & - & $1.00 \pm 0.00$ \\
\hline \multicolumn{7}{|c|}{ AuNP + nano-HAp } \\
\hline 1 month & $1.70 \pm 1.00$ & $0.00 \pm 0.00$ & $0.35 \pm 0.05$ & $1.00 \pm 0.00$ & $1.00 \pm 0.00$ & $1.00 \pm 0.00$ \\
\hline 3 months & $1.75 \pm 0.85$ & $0.20 \pm 0.20$ & $0.55 \pm 0.35$ & $2.00 \pm 0.00$ & 2.00 & $1.00 \pm 0.00$ \\
\hline 6 months & $1.91 \pm 0.24$ & $0.13 \pm 0.13$ & $1.06 \pm 0.13$ & $2.33 \pm 0.33$ & - & $1.67 \pm 0.33$ \\
\hline
\end{tabular}

Scores are given as mean \pm standard error of the mean. $P<0.05$.

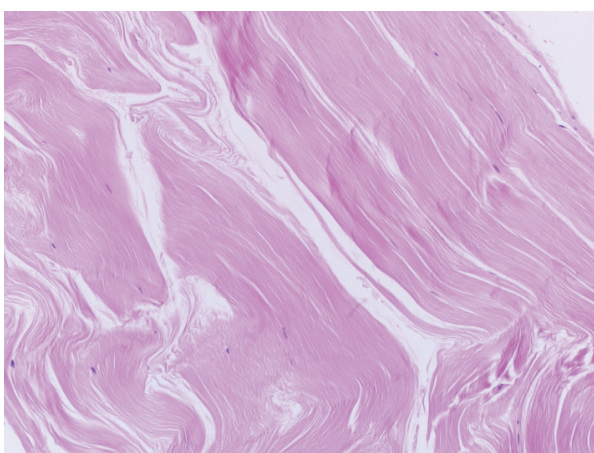

(a)

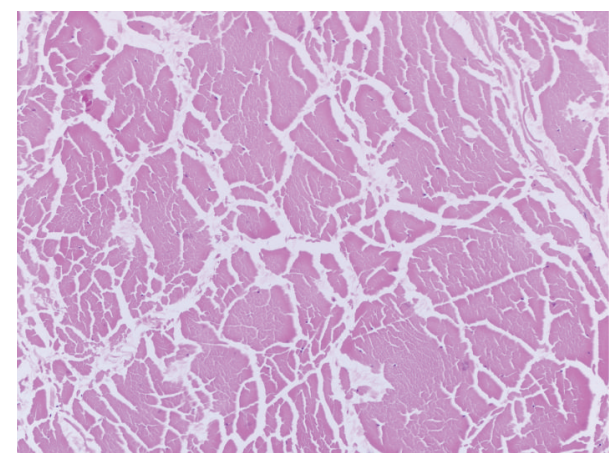

(b)

FIGURE 1: Control histology images of cross-linked human anterior tibialis tendon derived scaffolds before implantation demonstrating the heterogeneity of the scaffold between (a) the proximal end and (b) the distal end of the scaffold. Images are taken at 20x magnification.
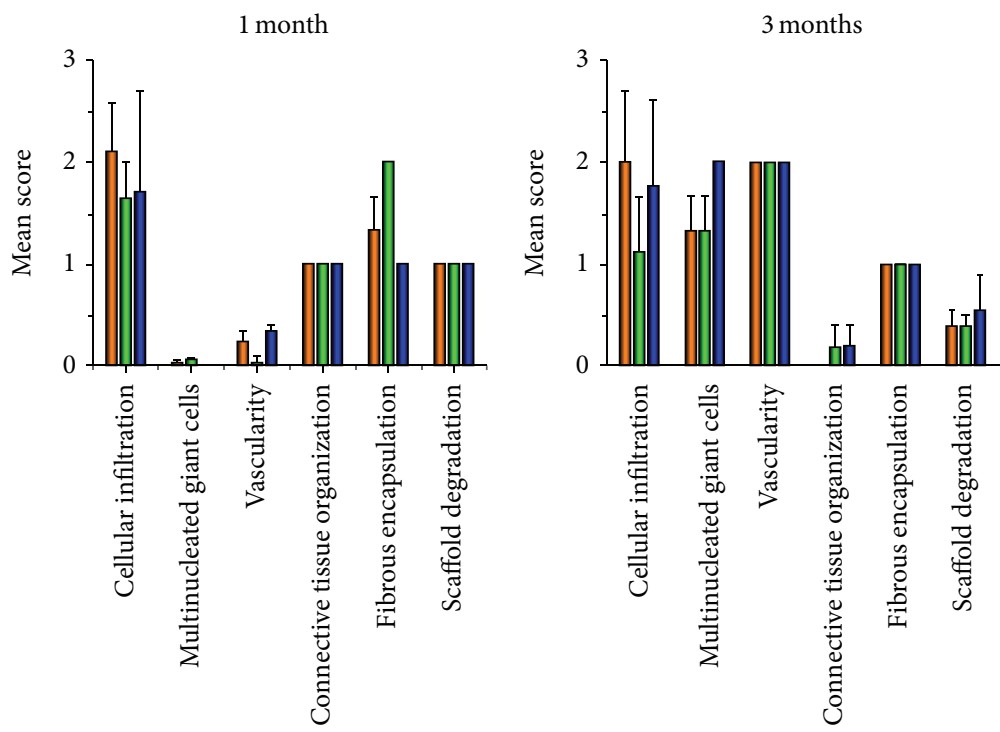
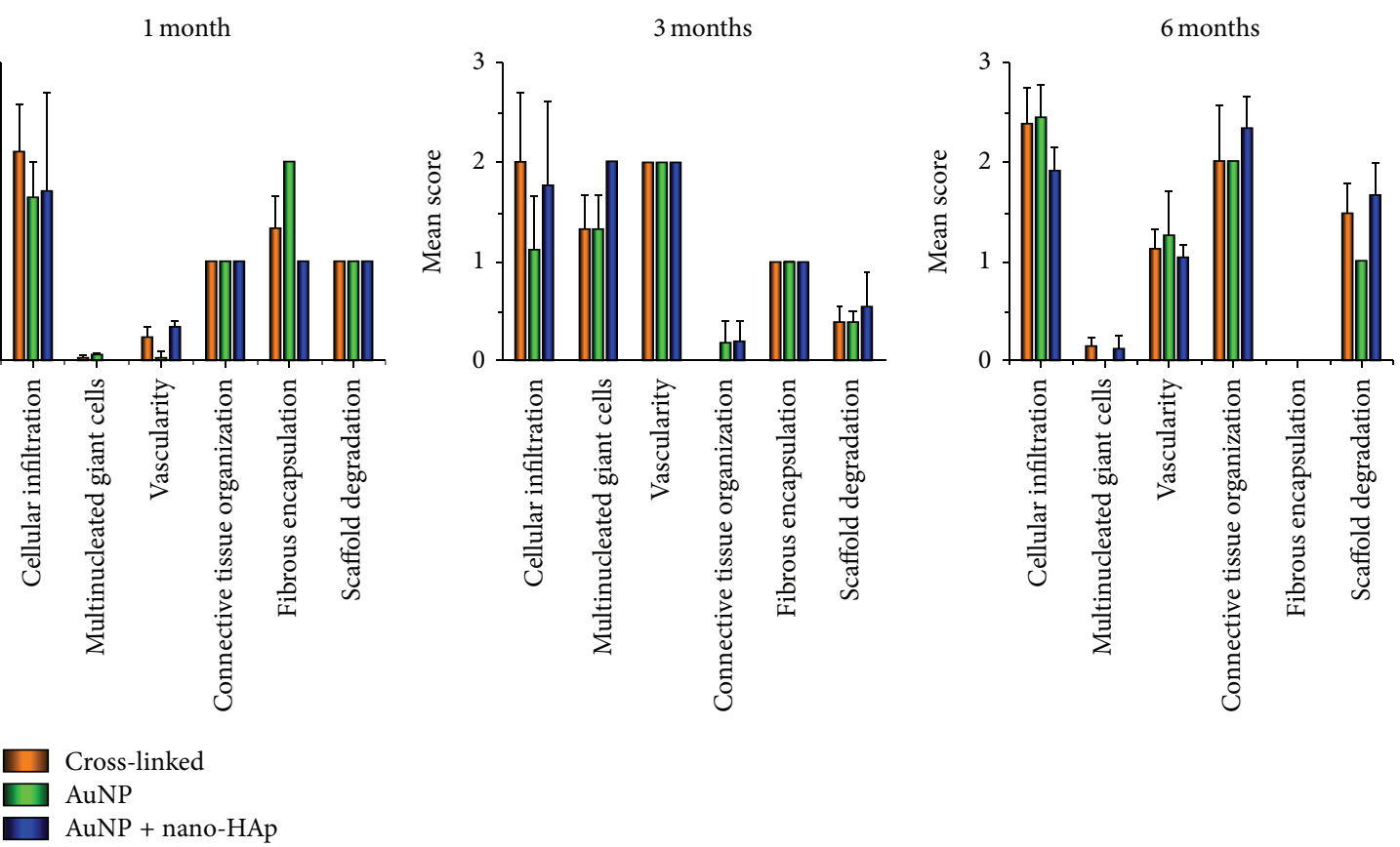

FIGURE 2: Semiquantitative and qualitative scores for all scaffolds at all time points. Mean scores are given as mean \pm standard error of the mean. $P<0.05$. 

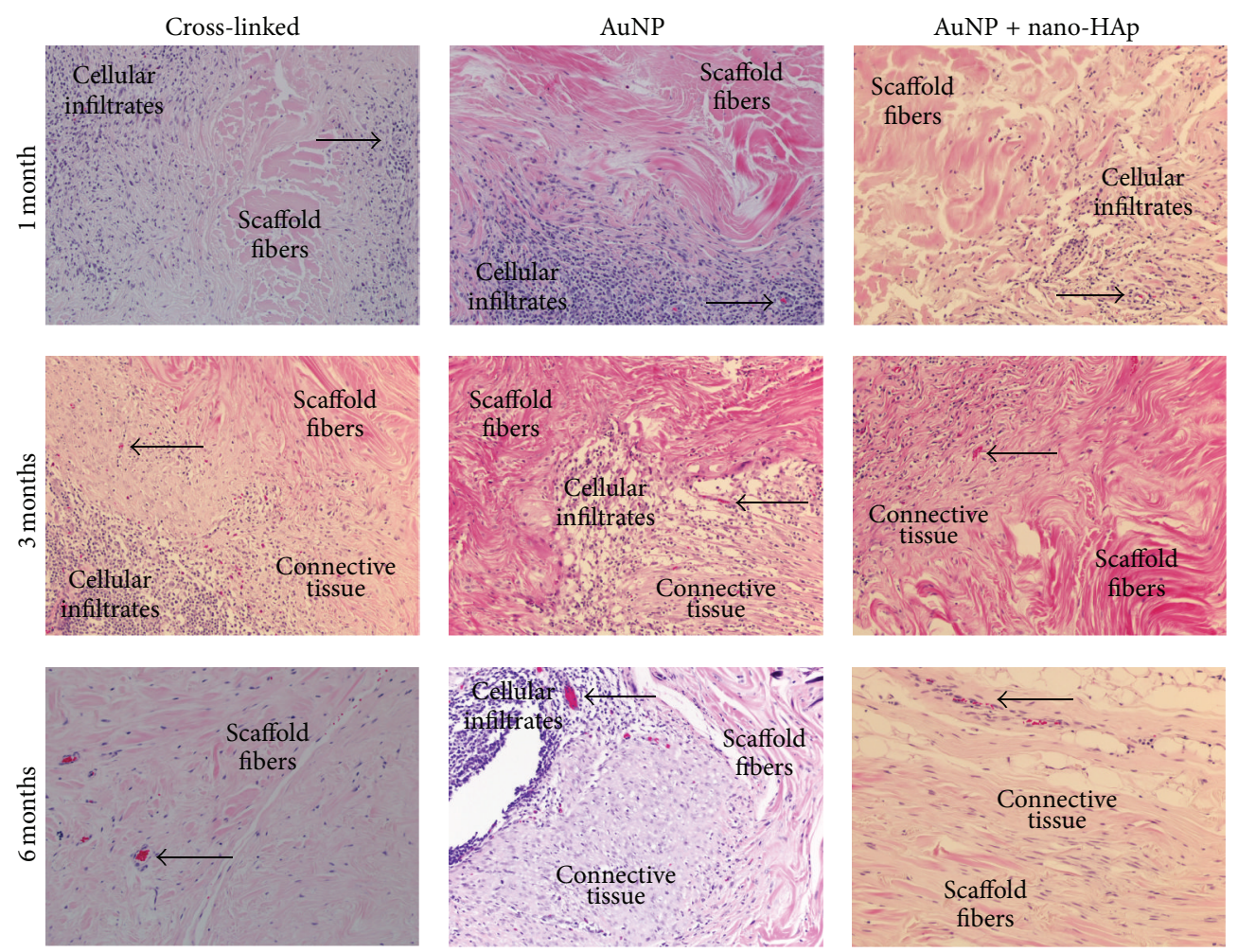

FIGURE 3: Histology images of H\&E stained explanted scaffolds taken at 20x. Arrows highlight blood vessels in the sample. Increases in cellular infiltration and connective tissue can be seen from 1 to 6 months.

not statistically significant, AuNP + nano-HAp scaffolds also show a similar trend of higher scores at 6 months compared to 1 and 3 months. At 6 months AuNP have the highest score. For all groups the increase from 1 month to 3 months is slight with a larger increase between 3 and 6 months. No significant differences are seen between groups at any time point.

For AuNP and AuNP + nano-HAp scaffolds, connective tissue organization scores are significantly higher at 6 months compared to 1 month. No significant differences are seen between groups at any time point but AuNP + nano-HAp scaffolds have the highest score at 6 months. All scaffolds have a score of 1 at 1 month which trends upwards at 3 and 6 months. At 1 month there is little host connective tissue infiltration within the scaffolds. At 3 months there is mild to moderate tissue infiltration. At 6 months there is moderate to abundant host connective tissue infiltration into the scaffolds.

Only one graft could be scored for fibrous encapsulation for cross-linked and AuNP + HAp scaffolds at 3 months. For cross-linked and AuNP + nano-HAp scaffolds, the score at 3 months is higher than at 1 month. Fibrous encapsulation was not scored at 6 months because the scaffold-subcutaneous layer could not be recovered during explantation. At 1 month AuNP scaffolds have significantly higher scores for fibrous encapsulation compared to scaffolds with AuNP + nanoHAp. At 1 month there is a mix of partially and mostly encapsulated scaffolds. At 3 months all scored scaffolds demonstrate some form of encapsulation but with incomplete borders.

No significant differences are seen between groups for scaffold degradation at any time point. Cross-linked scaffolds and AuNP + nano-HAp scaffolds have a score of 1 at 1 and 3 months with a slight increase at 6 months. AuNP scaffolds have a score of 1 at all time points and have the lowest score at 6 months. At 1 month most scaffolds have disrupted fibers that remain in large bundles or in a large central bundle. At 3 months scaffolds still show disruption but remain in large bundles. At 6 months scaffolds remain in large bundles with some small bundles and individual fibers present.

3.2. Qualitative Histology. A summary of sample images of $\mathrm{H} \& \mathrm{E}$ stained slides can be seen in Figure 3. At 1 month scaffolds appear largely intact and fibers remain together in bundles. There is mild to moderate cellular infiltration of mononuclear cells in the periphery of the scaffolds. There is little host connective tissue organization and no MNGC present. Several blood vessels are seen in the periphery of the scaffolds in the surrounding connective tissue. At 3 months scaffolds remain intact but show some disruption of fibers. Cellular infiltration is still mild to moderate with cell clusters at the periphery and center. There is mild to moderate host connective tissue organization and no MNGC are present. Slightly more blood vessels appear closer to the interface between the scaffold and connective tissue. At 6 months scaffolds remain in large bundles with some individual fibers seen. There is marked cellular infiltration of fibroblast cells throughout the scaffold. Abundant host connective tissue infiltration into the scaffold is present without any MNGC present. Blood vessels can be seen within and surrounding the scaffolds. Because these images are not taken at the subcutaneous layer interface, any evidence of 


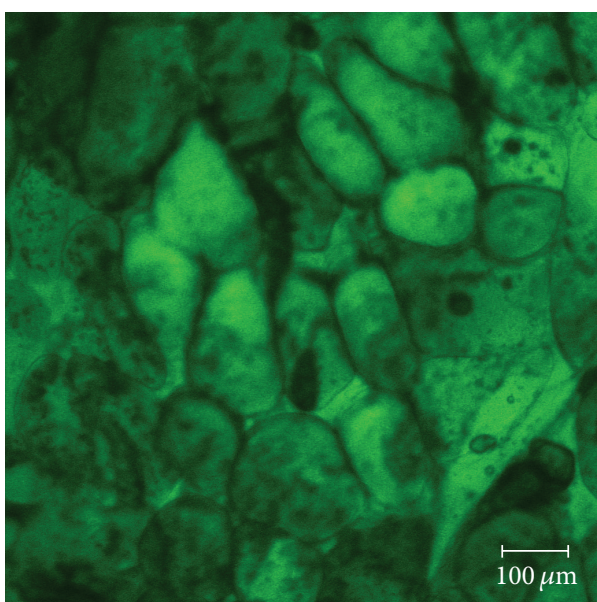

(a)

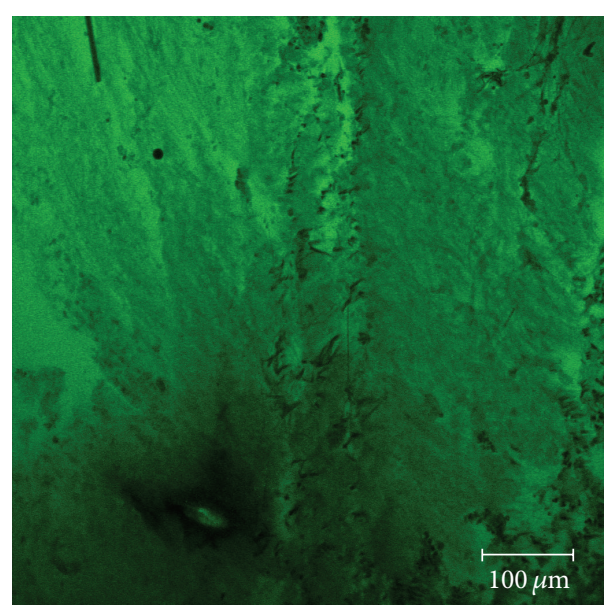

(b)

FIGURE 4: Control confocal microscope images of GFP swine tissue. (a) Tendon/muscle fibers and (b) connective tissue appear fluorescent.

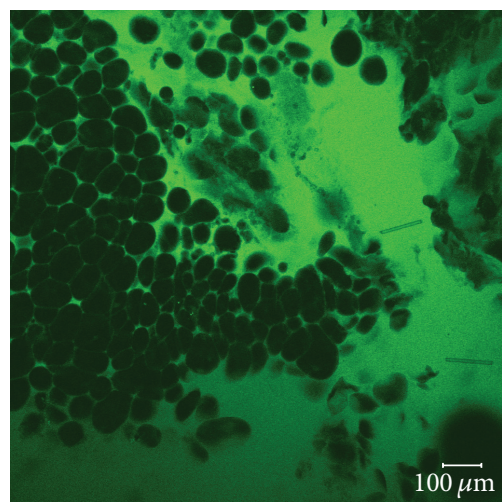

(a)

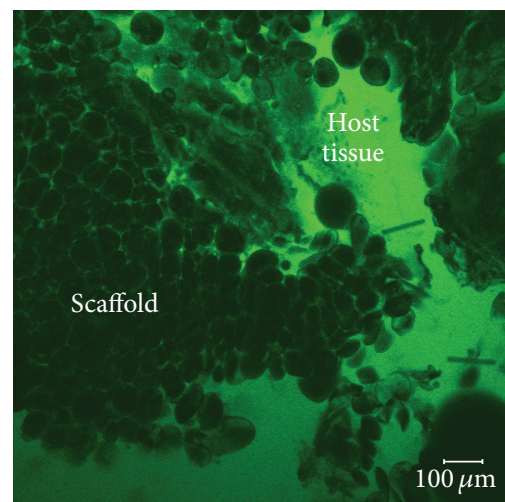

(b)

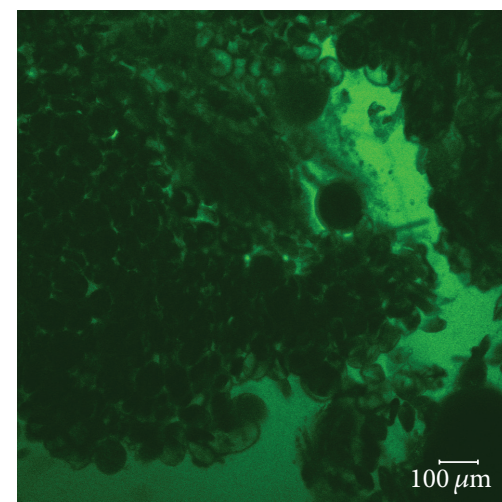

(c)

Figure 5: Slices of $z$-stacked confocal microscope images of an explanted AuNP scaffold at 1 month taken at (a) $15 \mu \mathrm{m}$, (b) $33 \mu \mathrm{m}$, and (c) $54 \mu \mathrm{m}$ relative distances within the sample. With each increasingly deeper slice, there appears to be less host tissue infiltration and bundles appear to be closer together.

fibrous encapsulation is not shown. Although not quantified, calcification was evident on all types of scaffolds at 6 months.

\subsection{Confocal Imaging. Images of GFP tissue harvested from} GFP expressing swine were acquired as a control to compare them to the explanted scaffolds. Confocal microscope images of control GFP tissue are shown in Figure 4. Figure 4(a) depicts tendon/muscle fibers of GFP tissue appearing fully fluorescent and mostly uniform. Figure 4(b) shows connective tissue also appearing fully fluorescent with an organized pattern. Some artifacts can be seen by small dark features but the tissue is mostly homogenous.

Confocal microscope images of explanted scaffolds and surrounding tissue show dark nonfluorescent scaffold bundles and fluorescent host tissue. Figure 5 shows 3 slices of a AuNP scaffold explanted at 1 month. Scaffold bundles are of uniform size with some fluorescent host tissue present between fibers. The images were taken as slices of a $z$-stacked image. Each slice was taken progressively deeper into the sample at $15 \mu \mathrm{m}$ (Figure 5(a)), $33 \mu \mathrm{m}$ (Figure 5(b)), and $54 \mu \mathrm{m}$
(Figure 5(c)) relative distances within the sample. With each increasingly deeper slice, there appears to be less host tissue infiltration and the bundles appear to be closer together. Figure 6 depicts confocal microscope images of cross-linked and AuNP scaffolds taken after 6 months. Green fluorescent host infiltration appears to be more distinct and fiber bundles appear to be more separated.

\section{Discussion}

The histology control images of the scaffold shown in Figure 1 provide an example of the need for improved methods to evaluate scaffold integration. ECM derived scaffold materials are often heterogeneous and may not be easily distinguished from host tissue. Scaffolds used in this study will fall on a spectrum of tissue organization between the two panels in Figure 1 depending on their location within the tissue.

Overall, human-derived scaffolds indicate good biocompatibility without a severe adverse host tissue response. Evidence of remodeling is present in the scaffolds which 


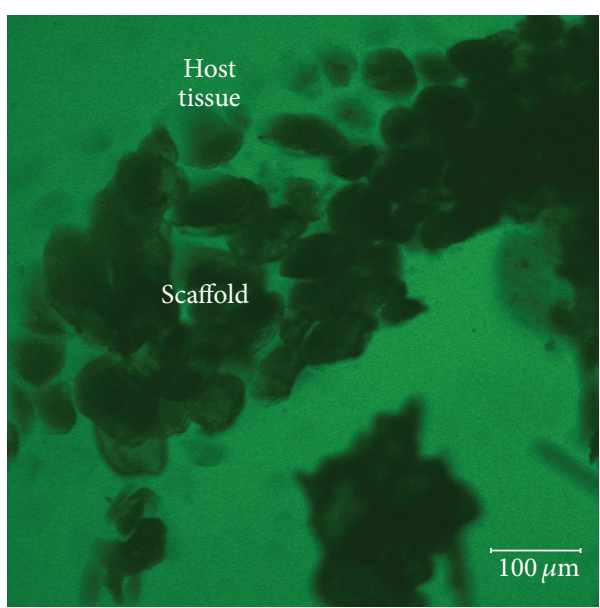

(a)

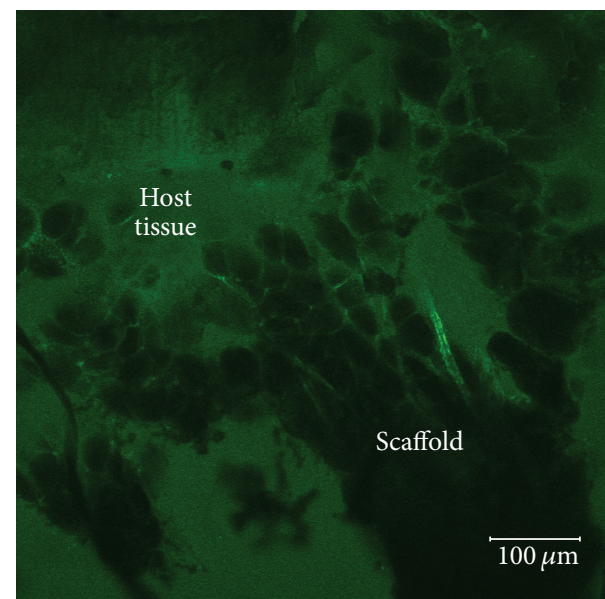

(b)

Figure 6: Confocal microscope images of explanted scaffolds at 6 months. (a) Cross-linked and (b) AuNP scaffolds show evidence of integration with scaffold bundles appearing to break apart slightly as host tissue infiltrates scaffold fibers. Laser power and imaging parameters were the same for both images.

includes cellular infiltration accompanied by blood vessels and new host connective tissue [37]. All scaffolds show evidence of increased fibroblast infiltration, vascularity, and connective tissue organization from 1 to 6 months. The combination of these properties indicates that the scaffold is being integrated and is not being rejected. Although there is evidence of fibrous encapsulation, it is not complete and is accompanied by signs of biocompatibility. If the scaffolds were to be completely encapsulated there would mostly likely be no evidence of cellular infiltration and vascularity [38]. Neovascularization is necessary to support cellular infiltration and new tissue growth which are both additional signs of biocompatibility. An increase in connective tissue organization also demonstrates that the host tissue is remodeling itself into a functionally organized tissue as opposed to disorganized scaffold remnants that would eventually be degraded. Organized connective tissue indicates that the host is restructuring and creating a stronger tissue to replace the scaffold. Very little evidence of MNGC supports that scaffolds are biocompatible. While large significant differences were not seen between groups with and without nanoparticles, the nanoparticle scaffolds demonstrated equal if not potentially better biocompatibility and remodeling behavior compared to control scaffolds. The lack of differences between scaffolds may be due to an insufficient amount of nanoparticles or delayed effects of the nanoparticles that are beyond the detection limits of this study.

Calcification is evident in histology images on all groups of scaffolds at 6 months. Calcification is marked by the accumulation of calcium deposited on or around the scaffold. There is not a correlation between calcification and a particular group of scaffolds including the scaffolds containing nanoHAp. Several cited causes of calcification include purity of the samples, type of animal model used, age of animal, fragmentation of elastin fibers, and high degrees of cross-linking [39-42]. Previous in vivo studies using scaffolds conjugated with nanoparticles did not show evidence of calcification and used scaffolds with similar purity, elastin fiber content, and degrees of cross-linking [30,31]. Key differences in this study compared to previous studies include the use of humanderived scaffolds in swine as opposed to porcine-derived scaffolds in swine [30]. Increased calcium content in scaffolds may increase calcification [42]. Scaffolds derived from human anterior tibialis tendon may be at an increased risk for calcium deposits because of its higher mineral content near the bone-tendon interface. Additionally, we have no reason to believe that the use of GFP expressing swine would induce an enhanced calcification response compared to standard swine models although this has not been investigated. It has been suggested that macrophage infiltration and inflammation may be precursors for calcification [43].

Confocal microscopy was utilized to examine the scaffold-host interface and identify host tissue migration into the scaffolds. There are several concerns and considerations that must be assessed in order to ascertain the validity of the images. The first consideration is the identification of the scaffold. The scaffold can be identified as the dark, nonfluorescent bundles against the bright green GFP tissue for several reasons (Figures 5 and 6). The structure can be interpreted from the size of the bundles in the images. The scaffold is derived from the human anterior tibialis whose bundles vary in size due to the structural differences proximally and distally. Tendon fibers can range between 30 and $200 \mathrm{~nm}$. These fibers are further bundled into groups of diameter $20 \mu \mathrm{m}, 20-200 \mu \mathrm{m}$, and eventually the tendon unit of $500 \mu \mathrm{m}$ which corresponds to the range of bundles seen in confocal images [44-46]. During imaging, the images were compared to a transmission channel to ensure that the dark features in the images were in fact from the tissue and not from empty space in the sample. In addition, the dark bundles evident in the images are most likely not tendon fibers from the host since host tendon fibers were observed to be fluorescent in control samples (Figure 4(a)). Lastly, nonimplanted control scaffolds, that is, fresh graft materials, 
were fluorescently imaged and no fluorescence signal was detected.

A second consideration in analyzing the confocal images is the presence of a green background in the images that arises from nonspecific GFP in the host tissue. As stated earlier, the host cells produce GFP. As host cells turnover, intracellular products including GFP are released into the surrounding tissue leading to a fluorescent extracellular space. Thus, a limitation to the GFP model is that it can be difficult to distinguish between individual host cells. The resulting fluorescence images (Figures 5 and 6) must be assumed to be fluorescent cells in a highly fluorescent extracellular space. In addition, it is imperative to ensure that fluorescence signals originate from the tissue samples and not from GFP leached into the PBS solution used for imaging. To mitigate this concern, we utilized the transmission channel on the microscope. Areas of host connective tissue that did not contain the scaffold were also imaged and did not have any dark bundles that could be interpreted as scaffold fibers (Figure 4(b)). This type of interpretation is consistent with the work of Higuchi et al. in which xenotransplanted cells in GFP mice were identified by GFP-negative areas [47].

Another consideration when observing the green host tissue integration is that GFP is very soluble in aqueous environments and therefore may leach from its original location into the surrounding tissue after harvesting [48]. Many of the studies citing problems of GFP leaching investigate intricate cellular processes and require detection of very small amounts of GFP. Studies of this type are much more sensitive to any type of GFP migration compared to ours. Thus small amounts of leaching are not a concern in this study due to the larger features of samples being imaged. In addition, there was a very large amount of GFP and fluorescence signal since a whole animal model was utilized. All parts of the host tissue produce and exhibit GFP in high amounts so that relative differences in amounts of GFP from migration cannot be distinguished by imaging techniques. Therefore, small amounts of GFP migration are not detectable and do not cause significant error when interpreting cell integration from the images.

The fluorescence microscopy images provide some unique qualitative information such as host tissue migration (Figure 5); however, it was difficult to acquire quantitative information for scaffold comparisons in this study. One way to quantify integration or host tissue response would be to quantify the fluorescence intensity of the host tissue integration into the scaffolds. This approach would require standardized reference points where the images would be acquired from the same location on each of the different scaffolds. It would require the same orientation of the scaffolds in reference to the host and the same depth of sectioning of the host-scaffold interface. This is a challenging but feasible endeavor that would require strict reference points during harvesting, freezing, sectioning, and imaging. In this study, we were unable to maintain clear reference points and were therefore unable to quantitatively compare test groups.

While quantitative data could not reliably be acquired, the fluorescence images provide supplementary qualitative data to histology results. The fluorescence images complement the results obtained from histological scoring. Evidence of cellular infiltration is seen in cross-sectional fluorescence images of explanted scaffold fibers as potential fluorescent cellular highways. Less host tissue is seen with progressively deeper images in Figure 5 which is consistent with histology findings of cellular infiltration and connective tissue organization remaining on the periphery of the scaffold at 1 month. Histology images indicate that although scaffolds are still mostly intact at 6 months, they appear to have fibers separated from the larger bundles (Figure 3). Similar results are seen in confocal microscope images (Figure 6) that depict a less organized and less uniform scaffold with evidence of host tissue infiltration between scaffold bundles at 6 months.

This study is the first report on the use of a GFP whole swine animal model to evaluate host tissue integration into soft tissue scaffolds conjugated with and without nanoparticles. While limitations in this study prevented the model from being a stand-alone method to evaluate host tissue integration, the model has potential for growth. Defining strict reference points during the harvesting, freezing, sectioning, and imaging would allow for quantitative comparisons between groups of specimens. In addition, as new GFP swine models develop, more specific GFP expression could aid in evaluating cellular integration as opposed to nonspecific general tissue integration [49]. With more specific labeling of the host cells and scaffolds, a quantitative approach could be taken similar to histological scoring. Another possible approach would be to utilize animal models with different types of fluorescent proteins such as red fluorescent protein [50-52]. In that method, the autofluorescence observed in the scaffold from chemical fixation methods or natural autofluorescence could be used to distinguish the scaffold from the host which would have a different emission spectrum. By developing a way to track cells and tag the scaffold, this model could have potential to provide additional quantitative data to evaluate host tissue integration.

\section{Conclusions}

Human-derived scaffolds were conjugated with AuNP and nano-HAp and implanted into GFP expressing swine for 1, 3 , and 6 months to evaluate biocompatibility and integration as well as to assess the use of GFP swine as a novel animal model. Qualitative and semiquantitative histology scores indicate potential remodeling of scaffolds from 1 to 6 months without significant differences between scaffolds with and without nanoparticles. Confocal microscope images complement histology results of cellular infiltration and host tissue integration. The use of GFP expressing swine provides qualitative data on host tissue integration and can be utilized as a supplement to support histology data. Future research in GFP swine models and scaffold labeling may improve this method of evaluation and allow it to be used as a way to quantitatively evaluate host tissue integration of soft tissue repair materials.

\section{Conflict of Interests}

The authors have no conflict of interests to declare. 


\section{Acknowledgments}

The authors would like to thank Aaron Wood, Allison Ostdiek, Scott Korte, Erin O'Connor, and Aleksandr Jurkevic for their contribution to this work. This material is based upon work supported by the National Science Foundation under Grant no. 0943941 and by the Coulter Translational Partnership Program at the University of Missouri.

\section{References}

[1] S. F. Badylak, D. O. Freytes, and T. W. Gilbert, "Extracellular matrix as a biological scaffold material: structure and function," Acta Biomaterialia, vol. 5, no. 1, pp. 1-13, 2009.

[2] S. F. Badylak, "Decellularized allogeneic and xenogeneic tissue as a bioscaffold for regenerative medicine: factors that influence the host response," Annals of Biomedical Engineering, vol. 42, no. 7, pp. 1517-1527, 2014.

[3] N. L. Leong, F. A. Petrigliano, and D. R. McAllister, "Current tissue engineering strategies in anterior cruciate ligament reconstruction," Journal of Biomedical Materials Research Part A, vol. 102, no. 5, pp. 1614-1624, 2014.

[4] L. Melman, E. D. Jenkins, N. A. Hamilton et al., "Early biocompatibility of crosslinked and non-crosslinked biologic meshes in a porcine model of ventral hernia repair," Hernia, vol. 15, no. 2, pp. 157-164, 2011.

[5] S. P. Zhong, Y. Z. Zhang, and C. T. Lim, "Tissue scaffolds for skin wound healing and dermal reconstruction," WIREs Nanomed Nanobi, vol. 2, pp. 510-525, 2010.

[6] B. N. Brown and S. F. Badylak, "Extracellular matrix as an inductive scaffold for functional tissue reconstruction," Translational Research, vol. 163, no. 4, pp. 268-285, 2014.

[7] X. Jiang, Z. Kalajzic, P. Maye et al., "Histological analysis of GFP expression in murine bone," Journal of Histochemistry \& Cytochemistry, vol. 53, no. 5, pp. 593-602, 2005.

[8] N. Tamamaki, Y. Yanagawa, R. Tomioka, J.-I. Miyazaki, K. Obata, and T. Kaneko, "Green fluorescent protein expression and colocalization with calretinin, parvalbumin, and somatostatin in the GAD67-GFP knock-in mouse," Journal of Comparative Neurology, vol. 467, no. 1, pp. 60-79, 2003.

[9] V. V. Artym and K. Matsumoto, "Imaging cells in three-dimensional collagen matrix," Current Protocols in Cell Biology, 2010.

[10] N. Billinton and A. W. Knight, "Seeing the wood through the trees: a review of techniques for distinguishing green fluorescent protein from endogenous autofluorescence," Analytical Biochemistry, vol. 291, no. 2, pp. 175-197, 2001.

[11] W. Baschong, R. Suetterlin, and R. Hubert Laeng, "Control of autofluorescence of archival formaldehyde-fixed, paraffinembedded tissue in confocal laser scanning microscopy (CLSM)," Journal of Histochemistry and Cytochemistry, vol. 49, no. 12, pp. 1565-1571, 2001.

[12] N. Zhu, D. Chapman, D. Cooper, D. J. Schreyer, and X. Chen, "X-ray diffraction enhanced imaging as a novel method to visualize low-density scaffolds in soft tissue engineering," Tissue Engineering Part C: Methods, vol. 17, no. 11, pp. 1071-1080, 2011.

[13] E. T. Curtis, S. Zhang, V. Khalilzad-Sharghi, T. Boulet, and S. F. Othman, "Magnetic resonance elastography methodology for the evaluation of tissue engineered construct growth," Journal of Visualized Experiments, no. 60, article no. e3618, 2012.

[14] O. Morin, A. Gillis, J. Chen et al., "Megavoltage cone-beam CT: system description and clinical applications," Medical Dosimetry, vol. 31, no. 1, pp. 51-61, 2006.
[15] E. Brown, J. Mantell, D. Carter, G. Tilly, and P. Verkade, "Studying intracellular transport using high-pressure freezing and correlative light electron microscopy," Seminars in Cell \& Developmental Biology, vol. 20, no. 8, pp. 910-919, 2009.

[16] T. Murakami and E. Kobayashi, "GFP-transgenic animals for in vivo imaging: rats, rabbits, and pigs," in In Vivo Cellular Imaging Using Fluorescent Proteins, R. M. Hoffman, Ed., Methods in Molecular Biology, pp. 177-189, 2012.

[17] Z. Liu, J. Song, Z. Wang et al., "Green fluorescent protein (GFP) transgenic pig produced by somatic cell nuclear transfer," Chinese Science Bulletin, vol. 53, no. 7, pp. 1035-1039, 2008.

[18] X. Yu, L. Wang, Z. Xia et al., "Modulation of host osseointegration during bone regeneration by controlling exogenous stem cell differentiation using a material approach," Biomaterials Science, vol. 2, no. 2, pp. 242-251, 2014.

[19] T. Zantop, T. W. Gilbert, M. C. Yoder, and S. F. Badylak, "Extracellular matrix scaffolds are repopulated by bone marrowderived cells in a mouse model of achilles tendon reconstruction," Journal of Orthopaedic Research, vol. 24, no. 6, pp. 1299 1309, 2006.

[20] Y. Bai, P.-F. Lee, H. C. Gibbs, K. J. Bayless, and A. T. Yeh, "Dynamic multicomponent engineered tissue reorganization and matrix deposition measured with an integrated nonlinear optical microscopy-optical coherence microscopy system," Journal of Biomedical Optics, vol. 19, no. 3, Article ID 036014, 2014.

[21] A. E. Loiselle, L. Wei, M. Faryad et al., "Specific biomimetic hydroxyapatite nanotopographies enhance osteoblastic differentiation and bone graft osteointegration," Tissue Engineering: Part A, vol. 19, no. 15-16, pp. 1704-1712, 2013.

[22] A. A. Sawyer, K. M. Hennessy, and S. L. Bellis, "Regulation of mesenchymal stem cell attachment and spreading on hydroxyapatite by RGD peptides and adsorbed serum proteins," Biomaterials, vol. 26, no. 13, pp. 1467-1475, 2005.

[23] S. A. Grant, C. S. Spradling, D. N. Grant et al., "Assessment of the biocompatibility and stability of a gold nanoparticle collagen bioscaffold," Journal of Biomedical Materials Research Part A, vol. 102, no. 2, pp. 332-339, 2014.

[24] Y. Zhou, Y. Kong, S. Kundu, J. D. Cirillo, and H. Liang, "Antibacterial activities of gold and silver nanoparticles against Escherichia coli and bacillus Calmette-Guérin," Journal of Nanobiotechnology, vol. 10, article 19, 2012.

[25] Ö. A. Kalaycı, F. B. Cömert, B. Hazer, T. Atalay, K. A. Cavicchi, and M. Cakmak, "Synthesis, characterization, and antibacterial activity of metal nanoparticles embedded into amphiphilic comb-type graft copolymers," Polymer Bulletin, vol. 65, no. 3, pp. 215-226, 2010.

[26] J. Siegel, K. Kolářová, V. Vosmanská, S. Rimpelová, J. Leitner, and V. Švorčík, "Antibacterial properties of green-synthesized noble metal nanoparticles," Materials Letters, vol. 113, pp. 59-62, 2013.

[27] P. Dauthal and M. Mukhopadhyay, "In-vitro free radical scavenging activity of biosynthesized gold and silver nanoparticles using Prunus armeniaca (apricot) fruit extract," Journal of Nanoparticle Research, vol. 15, article 1366, 2012.

[28] P. Ionita, F. Spafiu, and C. Ghica, "Dual behavior of gold nanoparticles, as generators and scavengers for free radicals," Journal of Materials Science, vol. 43, no. 19, pp. 6571-6574, 2008.

[29] Y. Zhang, H. He, W.-J. Gao, S.-Y. Lu, Y. Liu, and H.-Y. Gu, "Rapid adhesion and proliferation of keratinocytes on the gold colloid/chitosan film scaffold," Materials Science and Engineering: C, vol. 29, pp. 908-912, 2009. 
[30] S. A. Grant, C. R. Deeken, S. R. Hamilton, D. A. Grant, S. L. Bachman, and B. J. Ramshaw, "A comparative study of the remodeling and integration of a novel AuNP-tissue scaffold and commercial tissue scaffolds in a porcine model," Journal of Biomedical Materials Research Part A, vol. 101, no. 10, pp. 27782787, 2013.

[31] C. R. Deeken, M. Esebua, S. L. Bachman, B. J. Ramshaw, and S. A. Grant, "Assessment of the biocompatibility of two novel, bionanocomposite scaffolds in a rodent model," Journal of Biomedical Materials Research Part B: Applied Biomaterials, vol. 96, no. 2, pp. 351-359, 2011.

[32] C. R. Deeken, M. J. Cozad, S. L. Bachman, B. J. Ramshaw, and S. A. Grant, "Characterization of bionanocomposite scaffolds comprised of amine-functionalized single-walled carbon nanotubes crosslinked to an acellular porcine tendon," Journal of Biomedical Materials Research: Part A, vol. 96, no. 3, pp. 584594, 2011.

[33] C. R. Deeken, S. L. Bachman, B. J. Ramshaw, and S. A. Grant, "Characterization of bionanocomposite scaffolds comprised of mercaptoethylamine-functionalized gold nanoparticles crosslinked to acellular porcine tissue," Journal of Materials Science: Materials in Medicine, vol. 23, no. 2, pp. 537-546, 2012.

[34] C. R. Deeken, D. B. Fox, S. L. Bachman, B. J. Ramshaw, and S. A. Grant, "Characterization of bionanocomposite scaffolds comprised of amine-functionalized gold nanoparticles and silicon carbide nanowires crosslinked to an acellular porcine tendon," Journal of Biomedical Materials Research Part B: Applied Biomaterials, vol. 97, no. 2, pp. 334-344, 2011.

[35] M. J. Cozad, S. L. Bachman, and S. A. Grant, "Assessment of decellularized porcine diaphragm conjugated with gold nanomaterials as a tissue scaffold for wound healing," Journal of Biomedical Materials Research Part A, vol. 99, no. 3, pp. 426-434, 2011.

[36] J. E. Valentin, J. S. Badylak, G. P. McCabe, and S. F. Badylak, "Extracellular matrix bioscaffolds for orthopaedic applications: a comparative histologic study," Journal of Bone and Joint Surgery: Series A, vol. 88, no. 12, pp. 2673-2686, 2006.

[37] L. Pauzenberger, S. Syré, and M. Schurz, "Ligamentization' in hamstring tendon grafts after anterior cruciate ligament reconstruction: a systematic review of the literature and a glimpse into the future," Arthroscopy, vol. 29, no. 10, pp. 1712-1721, 2013.

[38] J. M. Anderson, A. Rodriguez, and D. T. Chang, "Foreign body reaction to biomaterials," Seminars in Immunology, vol. 20, no. 2, pp. 86-100, 2008.

[39] G. Golomb, F. J. Schoen, M. S. Smith, J. Linden, M. Dixon, and R. J. Levy, "The role of glutaraldehyde-induced cross-links in calcification of bovine pericardium used in cardiac valve bioprostheses," The American Journal of Pathology, vol. 127, no. 1, pp. 122-130, 1987.

[40] M. T. Bailey, S. Pillarisetti, H. Xiao, and N. R. Vyavahare, "Role of elastin in pathologic calcification of xenograft heart valves," Journal of Biomedical Materials Research Part A, vol. 66, no. 1, pp. 93-102, 2003.

[41] A. Singla and C. H. Lee, "Effect of elastin on the calcification rate of collagen-elastin matrix systems," Journal of Biomedical Materials Research, vol. 60, no. 3, pp. 368-374, 2002.

[42] W. F. Daamen, S. T. M. Nillesen, T. Hafmans, J. H. Veerkamp, M. J. A. van Luyn, and T. H. van Kuppevelt, "Tissue response of defined collagen-elastin scaffolds in young and adult rats with special attention to calcification," Biomaterials, vol. 26, no. 1, pp. 81-92, 2005.
[43] S. E. P. New and E. Aikawa, "Role of extracellular vesicles in de novo mineralization: an additional novel mechanism of cardiovascular calcification," Arteriosclerosis, Thrombosis, and Vascular Biology, vol. 33, no. 8, pp. 1753-1758, 2013.

[44] K. Shino, B. W. Oakes, S. Horibe, K. Nakata, N. Nakamura, and P. A. Indelicato, "Collagen fibril populations in human anterior cruciate ligament allografts. Electron microscopic analysis," The American Journal of Sports Medicine, vol. 23, no. 2, pp. 203-209, 1995.

[45] D. W. Jackson, J. Corsetti, and T. M. Simon, "Biologic incorporation of allograft anterior cruciate ligament replacements," Clinical Orthopaedics and Related Research, no. 324, pp. 126-133, 1996.

[46] U. Bosch, B. Decker, H. D. Moller, W. J. Kasperczyk, and H. J. Oestern, "Collagen fibril organization in the patellar tendon autograft after posterior cruciate ligament reconstruction. A quantitative evaluation in a sheep model," The American Journal of Sports Medicine, vol. 23, no. 2, pp. 196-202, 1995.

[47] Y. Higuchi, K. Kawai, M. Yamamoto et al., "A novel enhanced green fluorescent protein-expressing NOG mouse for analyzing the microenvironment of xenograft tissues," Experimental Animals, vol. 63, no. 1, pp. 55-62, 2014.

[48] H. Jockusch, S. Voigt, and D. Eberhard, "Localization of GFP in frozen sections from unfixed mouse tissues: immobilization of a highly soluble marker protein by formaldehyde vapor," Journal of Histochemistry \& Cytochemistry, vol. 51, no. 3, pp. 401-404, 2003.

[49] J. J. Whyte and R. S. Prather, "Genetic modifications of pigs for medicine and agriculture," Molecular Reproduction and Development, vol. 78, no. 10-11, pp. 879-891, 2011.

[50] Y. Lu, J.-D. Kang, S. Li et al., "Generation of transgenic Wuzhishan miniature pigs expressing monomeric red fluorescent protein by somatic cell nuclear transfer," Genesis, vol. 51, no. 8, pp. 575-586, 2013.

[51] H. Matsunari, M. Onodera, N. Tada et al., “Transgenic-cloned pigs systemically expressing red fluorescent protein, KusabiraOrange," Cloning and Stem Cells, vol. 10, no. 3, pp. 313-323, 2008.

[52] N. L. Webster, M. Forni, M. L. Bacci et al., "Multi-transgenic pigs expressing three fluorescent proteins produced with high efficiency by sperm mediated gene transfer," Molecular Reproduction and Development, vol. 72, no. 1, pp. 68-76, 2005. 


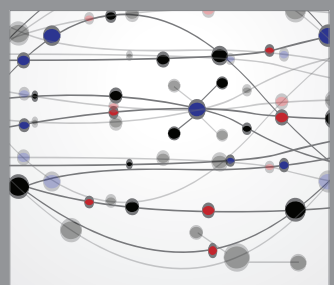

The Scientific World Journal
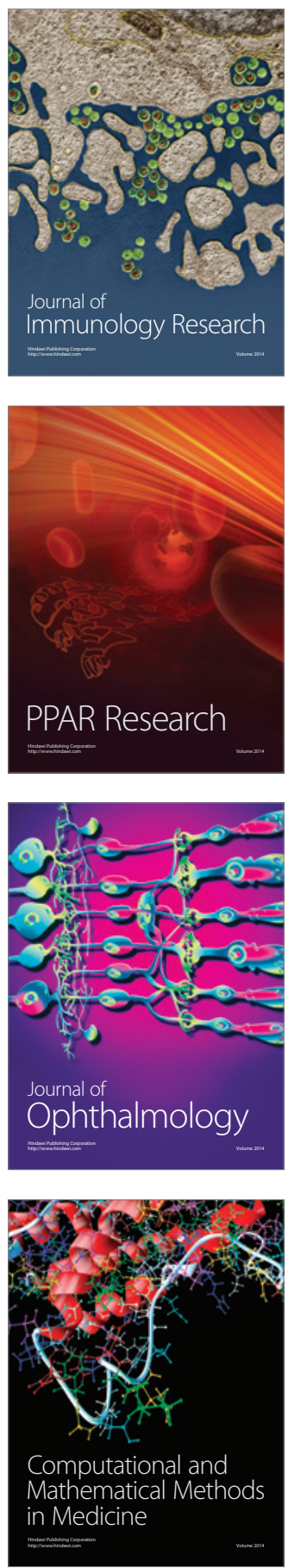

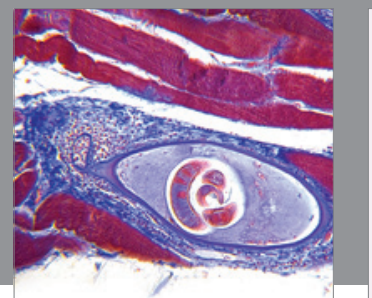

Gastroenterology

Research and Practice
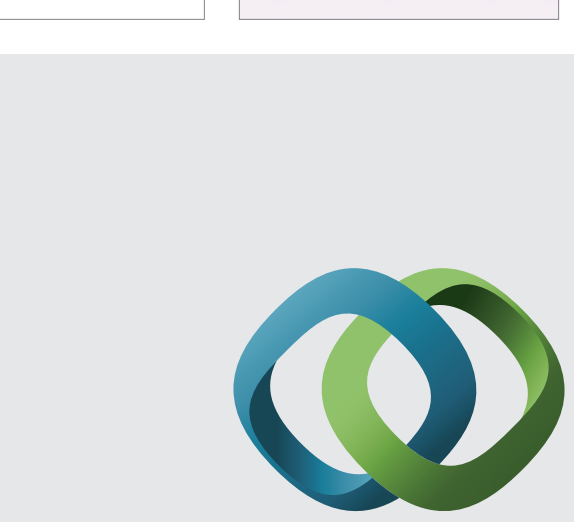

\section{Hindawi}

Submit your manuscripts at

http://www.hindawi.com
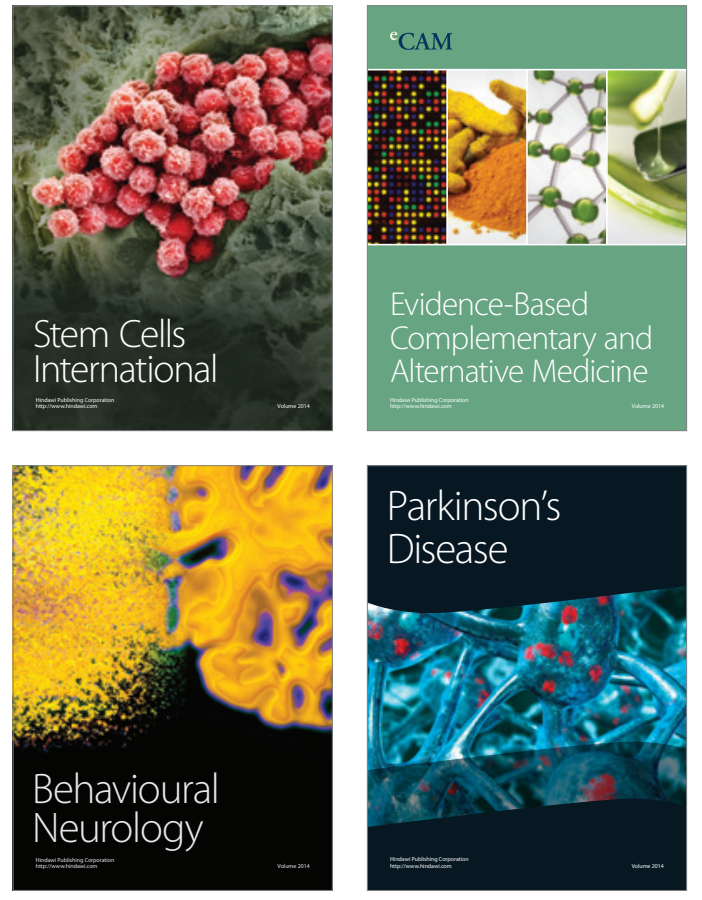
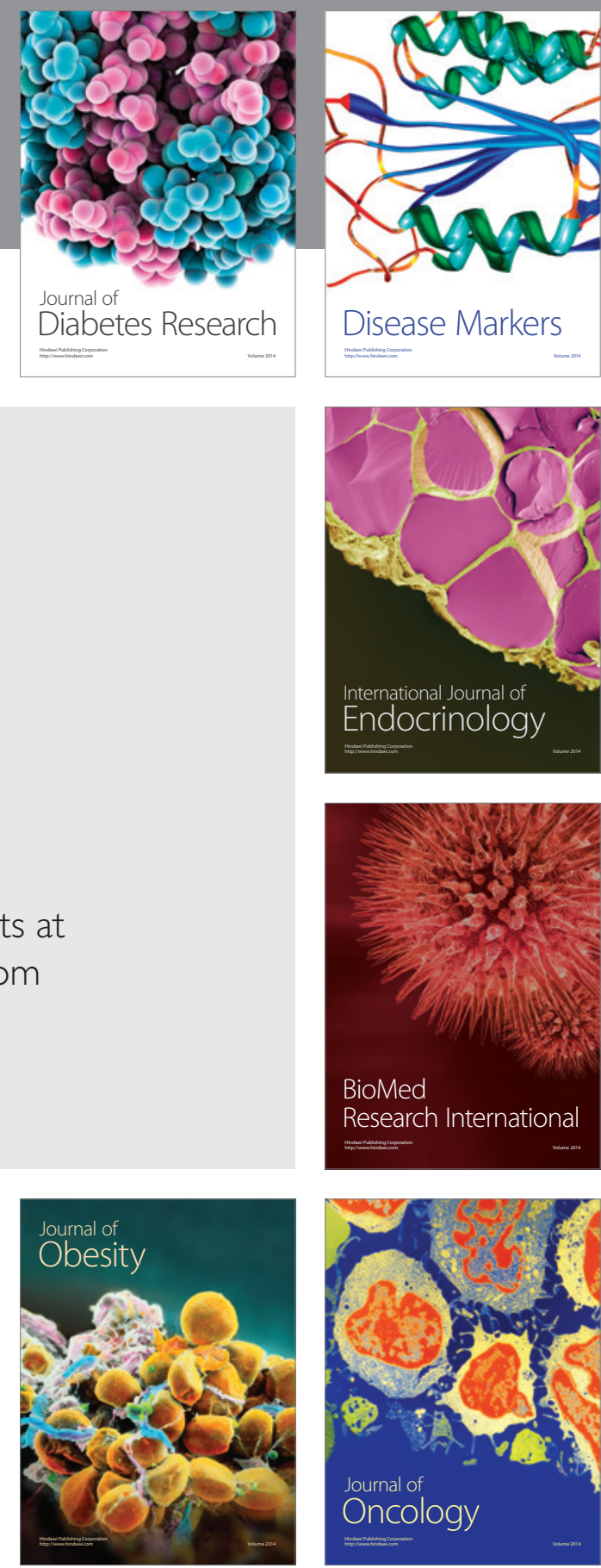

Disease Markers
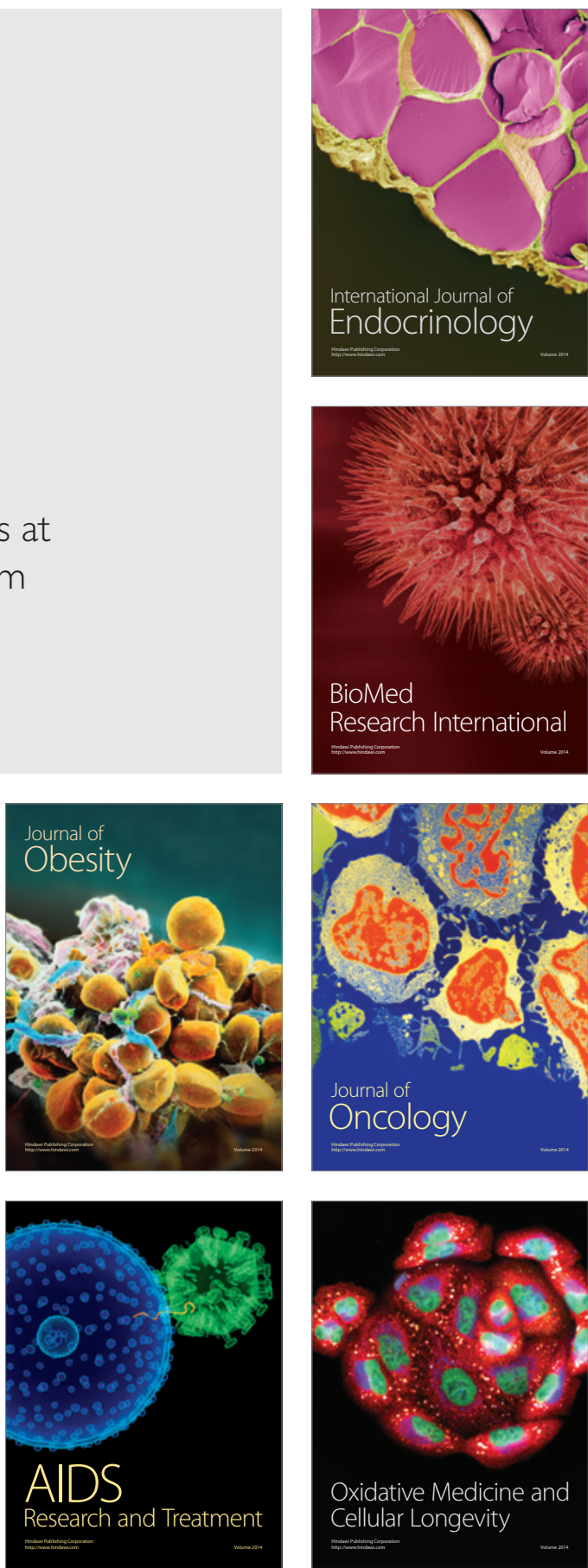